\title{
Size and Shape of Protein Molecules at the Nanometer Level Determined by Sedimentation, Gel Filtration, and Electron Microscopy
}

\author{
Harold P. Erickson
}

\begin{abstract}
An important part of characterizing any protein molecule is to determine its size and shape. Sedimentation and gel filtration are hydrodynamic techniques that can be used for this medium resolution structural analysis. This review collects a number of simple calculations that are useful for thinking about protein structure at the nanometer level. Readers are reminded that the Perrin equation is generally not a valid approach to determine the shape of proteins. Instead, a simple guideline is presented, based on the measured sedimentation coefficient and a calculated maximum $S$, to estimate if a protein is globular or elongated. It is recalled that a gel filtration column fractionates proteins on the basis of their Stokes radius, not molecular weight. The molecular weight can be determined by combining gradient sedimentation and gel filtration, techniques available in most biochemistry laboratories, as originally proposed by Siegel and Monte. Finally, rotary shadowing and negative stain electron microscopy are powerful techniques for resolving the size and shape of single protein molecules and complexes at the nanometer level. A combination of hydrodynamics and electron microscopy is especially powerful.
\end{abstract}

Keywords: Protein shape, hydrodynamics, gel filtration, sedimentation, electron microscopy.

\section{Introduction}

Most proteins fold into globular domains. Protein folding is driven largely by the hydrophobic effect, which seeks to minimize contact of the polypeptide with solvent. Most proteins fold into globular domains, which have a minimal surface area. Peptides from 10 to $30 \mathrm{kDa}$ typically fold into a single domain. Peptides larger than $50 \mathrm{kDa}$ typically form two or more domains that are

Shulin Li (ed.), Biological Procedures Online, Volume 11, Number 1

(C) to the author(s) 2009

DOI: 10.1007/s12575-009-9008-x URL: springerprotocols.com; springerlink.com 
independently folded. However, some proteins are highly elongated, either as a string of small globular domains or stabilized by specialized structures such as coiled coils or the collagen triple helix. The ultimate structural understanding of a protein comes from an atomic-level structure obtained by X-ray crystallography or nuclear magnetic resonance. However, structural information at the nanometer level is frequently invaluable. Hydrodynamics, in particular sedimentation and gel filtration, can provide this structural information, and it becomes even more powerful when combined with electron microscopy (EM).

One guiding principle enormously simplifies the analysis of protein structure. The interior of protein subunits and domains consists of closely packed atoms (1). There are no substantial holes and almost no water molecules in the protein interior. As a consequence of this, proteins are rigid structures, with a Young's modulus similar to that of Plexiglas (2). Engineers sometimes categorize biology as the science of "soft wet materials". This is true of some hydrated gels, but proteins are better thought of as hard dry plastic. This is obviously important for all of biology, to have a rigid material with which to construct the machinery of life. A second consequence of the close packed interior of proteins is that all proteins have approximately the same density, about $1.37 \mathrm{~g} / \mathrm{cm}^{3}$. For most of the following, we will use the partial specific volume, $v_{2}$, which is the reciprocal of the density. $v_{2}$ varies from 0.70 to 0.76 for different proteins, and there is a literature on calculating or determining the value experimentally. For the present discussion, we will ignore these variations and assume the average $v_{2}=$ $0.73 \mathrm{~cm}^{3} / \mathrm{g}$.

\section{How Big Is a Protein Molecule?}

Assuming this partial specific volume $\left(v_{2}=0.73 \mathrm{~cm}^{3} / \mathrm{g}\right)$, we can calculate the volume occupied by a protein of mass $M$ in Dalton as follows.

$$
\begin{aligned}
V\left(\mathrm{~nm}^{3}\right) & =\frac{\left(0.73 \mathrm{~cm}^{3} / \mathrm{g}\right) \times\left(10^{21} \mathrm{~nm}^{3} / \mathrm{cm}^{3}\right)}{6.023 \times 10^{23} \mathrm{Da} / \mathrm{g}} \times M(D a)[2.1] \\
& =1.212 \times 10^{-3}\left(\mathrm{~nm}^{3} / \mathrm{Da}\right) \times M(D a)
\end{aligned}
$$

The inverse relationship is also frequently useful: $M(\mathrm{Da})=$ $825 V\left(\mathrm{~nm}^{3}\right)$. 
What we really want is a physically intuitive parameter for the size of the protein. If we assume the protein has the simplest shape, a sphere, we can calculate its radius. We will refer to this as $R_{\min }$, because it is the minimal radius of a sphere that could contain the given mass of protein

$$
\begin{aligned}
R_{\min } & =(3 V / 4 \pi)^{1 / 3} \\
& =0.066 M^{1 / 3}\left(\text { for } M \text { in Dalton, } R_{\min } \text { in nanometer }\right) .
\end{aligned}
$$

Some useful examples for proteins from 5,000 to 500,000 Da are given in Table 1.

It is important to emphasize that this is the minimum radius of a smooth sphere that could contain the given mass of protein. Since proteins have an irregular surface, even ones that are approximately spherical will have an average radius larger than the minimum.

\section{How Far Apart Are Molecules in Solution?}

It is frequently useful to know the average volume of solution occupied by each molecule, or more directly, the average distance separating molecules in solution. This is a simple calculation based only on the molar concentration.

In a $1-M$ solution, there are $6 \times 10^{23}$ molecules $/ 1,=0.6$ molecules $/ \mathrm{nm}^{3}$, or inverting, the volume per molecule is $V=$ $1.66 \mathrm{~nm}^{3} /$ molecule at $1 \mathrm{M}$. For a concentration $C$, the volume per molecule is $V=1.66 / C$.

We will take the cube root of the volume per molecule as an indication of the average separation.

$$
d=V^{1 / 3}=1.18 / C^{1 / 3}
$$

where $C$ is in molar and $d$ is in nanometer. Table 2 gives some typical values.

\section{Table 1}

$\boldsymbol{R}_{\text {min }}$ for proteins of different mass

\begin{tabular}{llllllll} 
Protein $\boldsymbol{M}(\mathbf{k D a})$ & $\mathbf{5}$ & $\mathbf{1 0}$ & $\mathbf{2 0}$ & $\mathbf{5 0}$ & $\mathbf{1 0 0}$ & $\mathbf{2 0 0}$ & $\mathbf{5 0 0}$ \\
\hline$R_{\min }(\mathrm{nm})$ & 1.1 & 1.42 & 1.78 & 2.4 & 3.05 & 3.84 & 5.21 \\
\hline
\end{tabular}




\section{Table 2}

\section{Distance between molecules as function of concentration}

\begin{tabular}{lcccc} 
Concentration & $\mathbf{1 ~ M}$ & $\mathbf{1} \mathbf{~ m M}$ & $\mathbf{1} \mathbf{~ M}$ & $\mathbf{1 ~ n M}$ \\
\hline Distance between molecules $(\mathrm{nm})$ & 1.18 & 11.8 & 118 & 1,180 \\
\hline
\end{tabular}

Two interesting examples are hemoglobin and fibrinogen. Hemoglobin is $330 \mathrm{mg} / \mathrm{ml}$ in erythrocytes, making its concentration $0.005 \mathrm{M}$. The average separation of molecules (center to center) is $6.9 \mathrm{~nm}$. The diameter of a single hemoglobin molecule is about $5 \mathrm{~nm}$. These molecules are very concentrated, near the highest physiological concentration of any protein (the crystallins in lens cells can be at $>50 \%$ protein by weight).

Fibrinogen is a large rod-shaped molecule that forms a fibrin blood clot when activated. It circulates in plasma at a concentration of around $2.5 \mathrm{~g} / 1$, about $9 \mu \mathrm{M}$. The fibrinogen molecules are therefore about $60 \mathrm{~nm}$ apart, comparable to the 46-nm length of the rod-shaped molecule.

\section{The \\ Sedimentation \\ Coefficient and \\ Frictional Ratio. \\ Is the Protein \\ Globular or \\ Elongated?}

Biochemists have long attempted to deduce the shape of a protein molecule from hydrodynamic parameters. There are two major hydrodynamic methods that are used to study protein moleculessedimentation and diffusion (or gel filtration, which is the equivalent of measuring the diffusion coefficient).

The sedimentation coefficient, $S$, can be determined in an analytical ultracentrifuge. This was a standard part of the characterization of proteins in the 1940s and 1950s, and values of $S_{20, w}$ (sedimentation coefficient standardized to $20^{\circ} \mathrm{C}$ in water) are collected in references such as the Chemical Rubber Co. (CRC) Handbook of Biochemistry (3). Today, $S$ is more frequently determined by zone sedimentation in a sucrose or glycerol gradient, by comparison to standard proteins of known $S$. Five to twenty percent sucrose gradients have been most frequently used, but we prefer $15-40 \%$ glycerol gradients in $0.2 \mathrm{M}$ ammonium bicarbonate, because this is the buffer used for rotary shadowing EM (Section 6). The protein of interest is sedimented in one bucket of the swinging bucket rotor, and protein standards of known $S$ (Table 5) are sedimented in a separate (or sometimes the same) gradient. Following sedimentation, the gradient is eluted into fractions and each fraction is analyzed by sodium dodecyl sulfate polyacrylamide gel electrophoresis (SDS-PAGE) to locate the standards 
and the test protein. Figure 1 shows an example determining the sedimentation coefficient of the structural maintenance of chromosome (SMC) protein from Bacillus subtilis.

The sedimentation coefficient of a protein is a measure of how fast it moves through the gradient. Increasing the mass of the protein will increase its sedimentation, while increasing its size or asymmetry will decrease its sedimentation. The relationship of $S$ to size and shape of the protein is given by the Svedberg formula:

$$
S=M\left(1-v_{2} \rho\right) / N_{o} f=M\left(1-v_{2} \rho\right) /\left(N_{0} 6 \pi \eta R_{s}\right) .
$$

$M$ is the mass of the protein molecule in Dalton; $N_{\mathrm{o}}$ is Avogadro's number, $6.023 \times 10^{23} ; v_{2}$ is the partial specific volume of the protein; typical value is $0.73 \mathrm{~cm}^{3} / \mathrm{g}$; $\rho$ is the density of solvent $\left(1.0 \mathrm{~g} / \mathrm{cm}^{3}\right.$ for $\left.\mathrm{H}_{2} \mathrm{O}\right) ; \eta$ is the viscosity of the solvent $\left(0.01 \mathrm{~g} / \mathrm{cm}^{-\mathrm{s}}\right.$ for $\left.\mathrm{H}_{2} \mathrm{O}\right)$.

A critical factor in the equation is the frictional coefficient, $f$ (dimensions gram per second) which depends on both the size and shape of the protein. For a given mass of protein (or given volume), $f$ will increase as the protein becomes elongated or asymmetrical $(f$ can be replaced by an equivalent expression containing $R_{\mathrm{s}}$, the Stokes radius, to be discussed later). $S$ has the dimensions of time (seconds). For typical protein molecules, $S$ is in the range

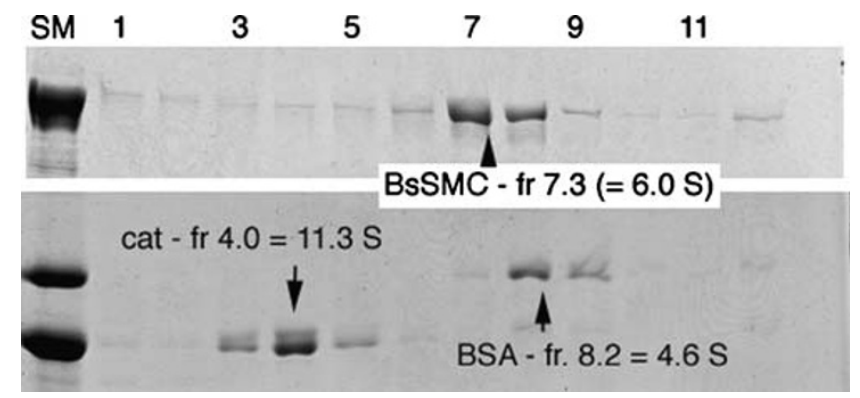

Fig. 1. Glycerol gradient sedimentation analysis of SMC protein from $B$. subtilis (BSSMC; upper panel) and sedimentation standards catalase and bovine serum albumin (lower pane). A $200-\mu \mathrm{l}$ sample was layered on a $5.0-\mathrm{ml}$ gradient of $15-40 \%$ glycerol in $0.2 \mathrm{M}$ ammonium bicarbonate and centrifuged in a Beckman SW55.1 swinging bucket rotor, $16 \mathrm{~h}, 38,000 \mathrm{rpm}$, $20^{\circ} \mathrm{C}$. Twelve fractions of $400 \mu \mathrm{l}$ each were collected from a hole in the bottom of the tube and each fraction was run on SDS-PAGE. Lane SM shows the starting material, and fraction 1 is the bottom of the gradient. The bottom panel shows that the 11.3-S catalase eluted precisely in fraction 4, while the 4.6-S BSA eluted mostly in fraction 8 , with some in fraction 9. We estimated the BSA to be centered on fraction 8.2. Experiments with additional standard proteins have demonstrated that the 15-40\% glycerol gradients are linear over the range 3-20 S, so a linear interpolation is used to determine $S$ of the unknown protein. BsSMC is in fractions 7 and 8, estimated more precisely at fraction 7.3. Extrapolating from the standards, we determine a sedimentation coefficient of $6.0 \mathrm{~S}$ for BsSMC. Other experiments gave an average value of $6.3 \mathrm{~S}$ for BsSMC (19). 
of $2-20 \times 10^{-13} \mathrm{~s}$, and the value $10^{-13} \mathrm{~s}$ is designated a Svedberg unit, $S$. Thus, typical proteins have sedimentation coefficients of 2-20 S.

From the above definition of parameters, it is clear that $S$ depends on the solvent and temperature. In classical studies, the solvent-dependent factors were eliminated and the sedimentation coefficient was extrapolated to the value it would have at $20^{\circ} \mathrm{C}$ in water (for which $\rho$ and $\eta$ are given above). This is referred to as $S_{20, w}$. In the present treatment, we will be referring mostly to standard proteins that have already been characterized, or unknown ones that will be referenced to these in gradient sedimentation, so our use of $S$ will always mean $S_{20, w}$.

A useful concept is the minimum value of $f$, which would obtain if the given mass of protein were packed into a smooth unhydrated sphere. As we have discussed in Section 1, the radius of this sphere will be $R_{\min }=0.066 M^{1 / 3}$ (Eq. 2.2). In about 1850, G. G. Stokes calculated theoretically the frictional coefficient of a smooth sphere (note that the equation is similar to that for the Stokes radius, to be discussed later, but the parameters here are different):

$$
f_{\min }=6 \pi \eta R_{\min }
$$

We have now designated $f_{\text {min }}$ as the minimal frictional coefficient for a protein of a given mass, which would obtain if the protein were a smooth sphere of radius $R_{\text {min }}$.

The actual $f$ of a protein will always be larger than $f_{\min }$ because of two things. First, the shape of the protein normally deviates from spherical, to be ellipsoidal or elongated; closely related to this is the fact that the surface of the protein is not smooth but rather rough on the scale of the water molecules it is traveling through. Second, all proteins are surrounded by a shell of bound water, one-two molecules thick, which is partially immobilized or frozen by contact with the protein. This water of hydration increases the effective size of the protein and thus increases $f$.

4.1. The Perrin Equation Does Not Work for Proteins
If one could determine the amount of water of hydration and factor this out, there would be hope that the remaining excess of $f$ over $f_{\min }$ could be interpreted in terms of shape. Algorithms have been devised for estimating the amount of bound water from the amino acid sequence, but these generally do not distinguish between buried residues, which have no bound water and surface residues which bind water. Some attempts have been made to base the estimate of bound water based on polar residues, which are mostly exposed on the surface. A $0.3-\mathrm{g} \mathrm{H}_{2} \mathrm{O} / \mathrm{g}$ protein is a typical estimate, but in fact, this kind of guess is almost useless for analyzing $f$. 
In the older days, when there was some confidence in these estimates of bound water, physical chemists calculated a value called $f_{\mathrm{o}}$, which was the frictional coefficient for a sphere that would contain the given protein, but enlarged by the estimated shell of water (other authors use $f_{\mathrm{o}}$ to designate what we term $f_{\min }$ $(3,4)$; we recommend using $f_{\min }$ to avoid ambiguity). The measured $f$ for proteins was almost always larger than $f_{\mathrm{o}}$, suggesting that the protein was asymmetrical or elongated. A very popular analysis was to model the protein as an ellipsoid of revolution and calculate the axial ratio from $f / f_{\mathrm{o}}$, using an equation first developed by Perrin. This approach is detailed in most classical texts of physical biochemistry. In fact, the Perrin analysis always overestimates the asymmetry of the proteins, typically by a factor of two to five. It should not be used for proteins.

The problem is illustrated by an early collaborative study of phosphofructokinase, in which the laboratory of James Lee did hydrodynamics and our laboratory did EM (5). We found by EM that the tetrameric particles were approximately cylinders, $9 \mathrm{~nm}$ in diameter and $14 \mathrm{~nm}$ long. The shape was therefore like a rugby ball, with an axial ratio of 1.5 for a prolate ellipsoid of revolution. The Lee group measured the molecular weight and sedimentation coefficient, determined $f$ and estimated water of hydration and $f_{\mathrm{o}}$. They then used the Perrin equation to calculate the axial ratio. The ratio was five, which would suggest that the protein had the shape of a hot dog. The EM structure (which was later confirmed by X-ray crystallography) shows that the Perrin equation overestimated the axial ratio by a factor of 3 .

Teller et al. (6) summarized the situation: "Frequently the axial ratios resulting from such treatment are absurd in light of the present knowledge of protein structure." They explained that the major problem with the Perrin equation is that it treats the protein as a smooth ellipsoid, when in fact the surface of the protein is quite rough. Teller et al. went on to show how the frictional coefficient can actually be derived from the known atomic structure of the protein, by modeling the surface of the protein as a shell of small beads of radius 1.4 $\AA$. The shell coated the surface of the protein, modeling its rugosity, and increasing the size of the protein by the equivalent of a single layer of bound water. This analysis has been extended by Garcia De La Torre and colleagues (7).

4.2. Interpreting Shape from $f / f_{\min }=S_{\max } / S$
If the Perrin equation is useless, is there some other way that shape can be interpreted from $f$ ? The answer is yes, at a semiquantitative level. We have discovered simple guidelines where the ratio $f / f_{\min }$ can provide a good indication of whether a protein is globular, somewhat elongated, or very elongated. 
Instead of proceeding with the classical ratio $f / f_{\min }$, where $f$ is in nonintuitive units, we will reformulate the analysis directly in terms of the sedimentation coefficient, which is the parameter actually measured. We will define a value $S_{\max }$ as the maximum possible sedimentation coefficient, corresponding to $f_{\min }$. $S_{\max }$ is the $S$ value that would be obtained if the protein were a smooth sphere with no bound water. These two ratios are equal: $f / f_{\min }=S_{\max } / S$. Combining Eqs. 2.2, 4.1, and 4.2, we have

$$
\begin{aligned}
S_{\max } & =10^{13} M\left(1-v_{2} \rho\right) / N_{o}\left(6 \pi \eta R_{\min }\right) \\
& =M\left[2.378 \times 10^{-4}\right] / R_{\min } \\
S_{\max } & =0.00361 M^{2 / 3} .
\end{aligned}
$$

The leading factor of $10^{13}$ in Eq. 4.3 a converts $S_{\max }$ to Svedberg units. The numbers in brackets in Eq. 4.3a are calculated using $v_{2}=0.73 \mathrm{~cm}^{3} / \mathrm{g}, \rho=1.0 \mathrm{~g} / \mathrm{cm}^{3}, \eta=0.01 \mathrm{~g} \mathrm{~cm}^{-1} \mathrm{~s}^{-1}=10^{-9} \mathrm{~g}$ $\mathrm{nm}^{-1} \mathrm{~s}^{-1}$. The final expression, Eq. $4.3 \mathrm{~b}$ expresses $S_{\max }$ in Svedbergs for a protein of mass $M$ in Daltons. Some typical numerical values of $S_{\max }$ for proteins from 10,000 to $1,000,000 \mathrm{Da}$ are given in Table 3.

We have surveyed values of $S_{\max } / S$ for a variety of proteins of known structure. Table 4 presents $S_{\max } / S$ for a number of approximately globular proteins and for a range of elongated proteins, all of known dimensions. It turns out that $S_{\max } / S$ is an excellent predictor of the degree of asymmetry of a protein. From this survey of known proteins, we can propose the following general principals.

- No protein has $S_{\max } / S=f / f_{\min }$ smaller than $\sim 1.2$.

- For approximately globular proteins: $S_{\max } / S$ is typically between 1.2 and 1.3 .

- For moderately elongated proteins: $S_{\max } / S$ is in the range of 1.5 to 1.9 .

- For highly elongated proteins (tropomyosin, fibrinogen, extended fibronectin):

$S_{\text {max }} / S$ is in the range of 2.0 to 3.0 .

Table 3

$S_{\max }$ calculated for proteins of different mass

\begin{tabular}{llllllll} 
Protein $\boldsymbol{M}_{\mathbf{r}} \mathbf{( k D a )}$ & $\mathbf{1 0}$ & $\mathbf{2 5}$ & $\mathbf{5 0}$ & $\mathbf{1 0 0}$ & $\mathbf{2 0 0}$ & $\mathbf{5 0 0}$ & $\mathbf{1 , 0 0 0}$ \\
\hline$S_{\max }$ Svedbergs & 1.68 & 3.1 & 4.9 & 7.8 & 12.3 & 22.7 & 36.1 \\
\hline
\end{tabular}




\section{Table 4}

\section{$S_{\max } / S$ values for representative globular and elongated proteins}

\begin{tabular}{|lccccc}
\hline Protein & Dimensions $(\mathbf{n m})$ & Mass & $\boldsymbol{S}_{\text {max }}$ & \multirow{S}{*}{$\boldsymbol{S}$} & $\boldsymbol{S}_{\text {max }} / \boldsymbol{S}$ \\
\hline Globular protein standards dimensions are from pdb files & & & & \\
\hline Phosphofructokinase & $14 \times 9 \times 9$ & 345,400 & 17.77 & 12.2 & 1.46 \\
\hline Catalase & $9.7 \times 9.2 \times 6.7$ & 230,000 & 13.6 & 11.3 & 1.20 \\
\hline Serum albumin & $7.5 \times 6.5 \times 4.0$ & 66,400 & 5.9 & 4.6 & 1.29 \\
\hline Hemoglobin & $6 \times 5 \times 5$ & 64,000 & 5.78 & 4.4 & 1.32 \\
\hline Ovalbumin & $7.0 \times 3.6 \times 3.0$ & 43,000 & 4.43 & 3.5 & 1.27 \\
\hline FtsZ & $4.8 \times 4 \times 3$ & 40,300 & 4.26 & 3.4 & 1.25 \\
\hline Elongated protein standards-tenascin fragments & $27,28) ;$ heat repeat $(29,30)$ & & \\
\hline TNfnl-5 & $14.7 \times 1.7 \times 2.8$ & 50,400 & 4.94 & 3.0 & 1.65 \\
\hline TNfnl-8 & $24.6 \times 1.7 \times 2.8$ & 78,900 & 6.64 & 3.6 & 1.85 \\
\hline TNfnALL & $47.9 \times 1.7 \times 2.8$ & 148,000 & 10.1 & 4.3 & 2.36 \\
\hline PR65/A HEAT repeat & $17.2 \times 3.5 \times 2.0$ & 60,000 & 5.53 & 3.6 & 1.54 \\
\hline Fibrinogen & $46 \times 3 \times 6$ & 390,000 & 19.3 & 7.9 & 2.44 \\
\hline
\end{tabular}

- For very long thread-like molecules like collagen, or huge extended molecules like the tenascin hexabrachion (not shown): $S_{\text {max }} / S$ can range from $3-4$ or more.

Apart from indicating the shape of a protein, $S_{\max } / S$ can often give valuable information about the oligomeric state, if one has some idea of the shape. For example, if one knows that the protein subunit is approximately globular (from EM for example), but finds $S_{\max } / S=2.1$, this would suggest that the protein in solution is actually a dimer. On the other hand, if one thinks a protein is a dimer, but finds $S_{\max } / S<1.0$ for the dimer mass, the protein is apparently sedimenting as a monomer.

The use of $S_{\max } / S$ to estimate protein shape has been described briefly in $(8)$.

\section{The Kirkwood/ Bloomfield Calculation}

The understanding of how protein shape affects hydrodynamics is elegantly extended by an analysis originally developed by Kirkwood (9) and later extended by Bloomfield and Garcia De La 
Torres (10-12). In its simplest application, it calculates the sedimentation coefficient of a rigid oligomeric protein composed of subunits of known $S$ and known spacing relative to each other. In more complex applications, a protein of any complex shape can be modeled as a set of nonoverlapping spheres or beads. See Byron (13) for a comprehensive review of the principals and applications of hydrodynamic bead modeling of biological macromolecules.

The basis of the Kirkwood/Bloomfield analysis is to account for how each bead shields the others from the effect of solvent flow and thereby determine the hydrodynamics of the ensemble from its component beads. Figure 2 shows a simple example of the bead modeling approach and provides an instructive look at how size and shape affect sedimentation. There are several important conclusions.

- A rod of three beads has about a twofold higher $S$ than a single bead.

- $S_{\max } / S$ is 1.18 for the single bead (the effect of the assumed shell of water), 1.34 for the three-bead rod, and 1.93 for the straight 11-bead rod. This is consistent with the principals given in Section 4 for globular, somewhat elongated, and very elongated particles.

- Bending the rod at $90^{\circ}$ in the middle causes only a small increase in $S$. Bending it into a $U$-shape with the arms about one bead diameter apart increases $S$ a bit more. Bending this same 11-bead structure more sharply, so the two arms are in contact, causes a substantial increase in $S$, from 5.05 to 5.58 . The guiding principle is that folding affects $S$ when one part of the molecule is brought close enough to another to shield it from water flow.

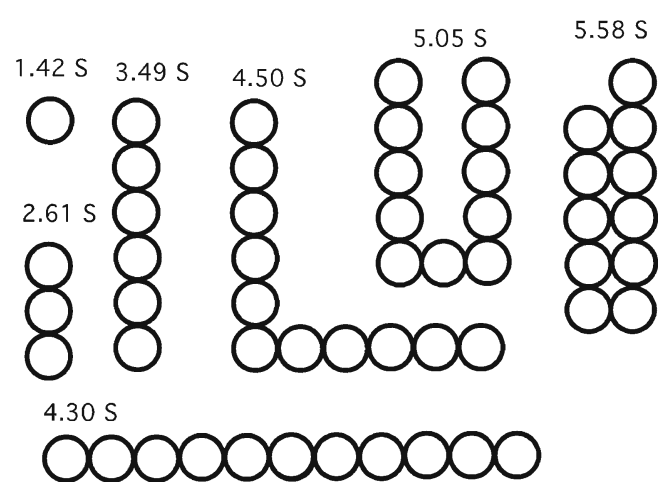

Fig. 2. Each bead models a 10-kDa domain, with an assumed sedimentation coefficient of $1.42 \mathrm{~S}$. The radius of the bead is $1.67 \mathrm{~nm}$, using $R_{\min }=$ $1.42 \mathrm{~nm}$, and adding $0.25 \mathrm{~nm}$ for a shell of water. The beads are an approximation to FN-III or Ig domains, which are $\sim 1.7 \times 2.8 \times 3.5 \mathrm{~nm}$. The sedimentation coefficients of multibead structures were calculated by the formula of Kirkwood/Bloomfield. 


\section{Gel Filtration Chromatography and the Stokes Radius}

"Gel filtration chromatography is widely used for determining protein molecular weight." This quote from Sigma-Aldrich bulletin 891A is a widely held misconception. The fallacy is obscurely corrected by a later note in the bulletin that "Once a calibration curve is prepared, the elution volume for a protein of similar shape, but unknown weight, can be used to determine the MW." The key issue is "of similar shape". Generally, the calibration proteins are all globular, and if the unknown protein is also globular, the calibrated gel filtration column does give a good approximation of its molecular weight. The problem is that the shape of an unknown protein is generally unknown. If the unknown protein is elongated, it can easily elute at a position twice the molecular weight of a globular protein.

The gel filtration column actually separates proteins not on their molecular weight but on their frictional coefficient. Since the frictional coefficient, $f$, is not an intuitive parameter, it is usually replaced by the Stokes radius $R_{\mathrm{s}}$. $R_{\mathrm{s}}$ is defined as the radius of a smooth sphere that would have the actual $f$ of the protein. This is much more intuitive since it allows one to imagine a real sphere approximately the size of the protein, or somewhat larger if the protein is elongated and has bound water.

As mentioned above for Eq. 4.2, Stokes calculated theoretically the frictional coefficient of a smooth sphere to be:

$$
f=6 \pi \eta R_{s} .
$$

The Stokes radius $R_{\mathrm{s}}$ is larger than $R_{\min }$ because it is the radius of a smooth sphere whose $f$ would match the actual $f$ of the protein. It accounts for both the asymmetry of the protein and the shell of bound water. More quantitatively, $f / f_{\min }=S_{\max } / S=R_{\mathrm{s}} / R_{\min }$.

Siegel and Monte (4) argued convincingly that the elution of proteins from a gel filtration column correlates closely with the Stokes radius, $R_{\mathrm{s}}$, presenting experimental data from a wide range of globular and elongated proteins. The Stokes radius is known for large number of proteins, including ones convenient for calibrating gel filtration columns (Table 5). Figure 3 shows an example where the $R_{\mathrm{s}}$ of the unknown protein SMC protein from $B$. subtilis was determined by gel filtration.

The standard proteins should span $R_{\mathrm{s}}$ values above and below that of the protein of interest (but in the case of SMC protein from B. subtilis, a short extrapolation to a larger value was used). The literature generally recommends determining the void and included volumes of the column and plotting a partition coefficient $K_{\mathrm{AV}}(4)$. However, we have found it generally satisfactory to sim- 


\section{Table 5}

\section{Standards for hydrodynamic analysis}

\begin{tabular}{lcccccc} 
Protein & $\boldsymbol{M}_{\mathbf{r}}$ aa seq & $\boldsymbol{S}_{\mathbf{2 0 , \mathbf { w }}}$ & $\boldsymbol{S}_{\text {max }} / \boldsymbol{S}$ & $\boldsymbol{R}_{\mathbf{s}}(\mathbf{n m})$ & Source & $\boldsymbol{M}_{\mathbf{r}}$ S-M \\
\hline Ribonuclease A beef pancreas & 14,044 & $2.0^{\mathrm{a}}$ & $1.05^{\mathrm{a}}$ & 1.64 & HBC & 13,791 \\
\hline Chymotrypsinogen A beef pancreas & 25,665 & 2.6 & 1.21 & 2.09 & HBC & 22,849 \\
\hline Ovalbumin hen egg & $42,910 \mathrm{~s}$ & 3.5 & 1.27 & 3.05 & HBC & 44,888 \\
\hline Albumin beef serum & 69,322 & $4.6^{\mathrm{a}}$ & 1.33 & 3.55 & $\mathrm{~S}-\mathrm{M}, \mathrm{HBC}$ & 68,667 \\
\hline Aldolase rabbit muscle & 157,368 & 7.3 & 1.45 & 4.81 & HBC & 147,650 \\
\hline Catalase beef liver & 239,656 & 11.3 & 1.21 & 5.2 & S-M & 247,085 \\
\hline Apo-ferritin horse spleen & 489,324 & 17.6 & 1.28 & 6.1 & HBC & 451,449 \\
\hline Thyroglobulin bovine & 606,444 & 19 & 1.37 & 8.5 & HBC & 679,107 \\
\hline Fibrinogen, human & 387,344 & 7.9 & 2.44 & 10.7 & S-M & 355,449 \\
\hline
\end{tabular}

Gel filtration calibration kits, containing globular proteins of known molecular weight and $R_{\mathrm{s}}$, are commercially available (GE Healthcare, Sigma-Aldrich). These same proteins can be used for sedimentation standards. The proteins in these kits are included in the table along with some others that we have found useful. The values for $M_{\mathrm{r}}$ given in the first column are from amino acid sequence data. Values for $S_{20, w}$ and $R_{\mathrm{s}}$ are from the Siegel-Monte paper (indicated S-M under source), or the CRC Handbook of Biochemistry (3) (indicated HBC). They agree with the values listed for $R_{\mathrm{s}}$ in the GE Healthcare gel filtration calibration kit, with the exception of ferritin. The " $M_{\mathrm{r}}$ calc" in the last column was obtained by our simplification of the Siegel-Monte calculation $\left(M=4,205 S R_{\mathrm{s}}\right)$. Note that the worst disagreement with " $M_{\mathrm{r}}$ aa seq" is about $10 \%$

${ }^{\text {a }} S$ for ribonuclease A is questionable because of the low $S_{\max } / S(1.05) . S$ values for bovine serum albumin vary in the literature from 4.3 to 4.9 . Many sources use 4.3 , but we find that 4.6 gives a better fit with other standards (note that the standard curve in Fig. 5 used 4.3, but 4.6 would have placed it closer to the line)

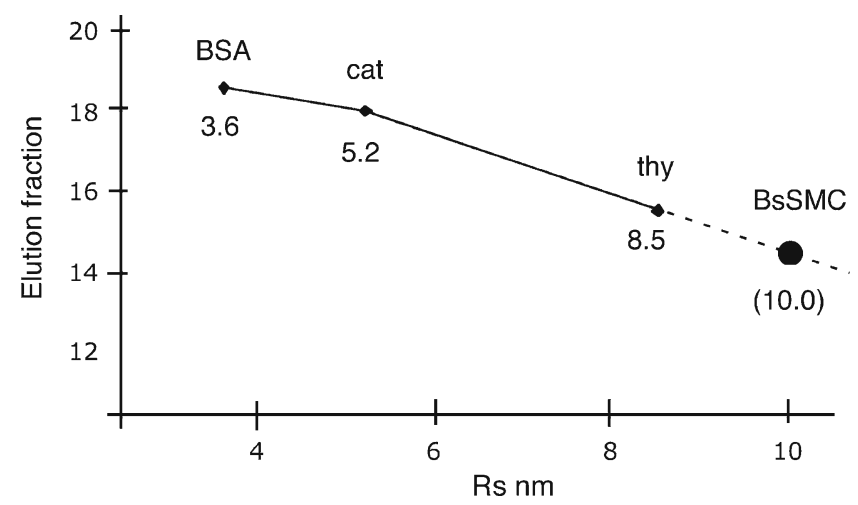

Fig. 3. Determination of $R_{\mathrm{s}}$ of BsSMC by gel filtration. The column was calibrated by running standard proteins BSA, catalase, and thyroglobulin over the column, then BsSMC. BsSMC eluted in fraction 14.2, which corresponds to an $R_{\mathrm{S}}$ of $10 \mathrm{~nm}$ on the extrapolated curve. In repeated experiments, the average $R_{\mathrm{s}}$ was $10.3 \mathrm{~nm}(19)$. 
ply plot elution position vs $R_{\mathrm{s}}$ for the standard proteins. This generally gives an approximately linear plot, but otherwise, it is satisfactory to draw lines between the points and read the $R_{\mathrm{s}}$ of the protein of interest from its elution position on this standard curve.

A gel filtration column can determine $R_{\mathrm{s}}$ relative to the $R_{\mathrm{s}}$ of the standard calibration proteins. The $R_{\mathrm{s}}$ of these standards was generally determined from experimentally measured diffusion coefficients. Some tabulations of hydrodynamic data list the diffusion coefficient, $D$, rather than $R_{\mathrm{s}}$, so it is worth knowing the relationship:

$$
D=k T / f=k T /\left(6 \pi \eta R_{s}\right) .
$$

where $k=1.38 \times 10^{-16} \mathrm{~g} \mathrm{~cm}^{2} \mathrm{~s}^{-2} \mathrm{~K}^{-1}$ is Boltzman's constant and $T$ is the absolute temperature. $k$ is given here in centimeter-gramsecond units because $D$ is typically expressed in centimetergram-second; $R_{s}$ will be expressed in centimeter in this equation. Typical proteins have $D$ in the range of $10^{-6}$ to $10^{-7} \mathrm{~cm}^{2} \mathrm{~s}^{-1}$. Converting to nanometer and for $T=300 \mathrm{~K}$ and $\eta=0.01$ :

$$
R_{s}=(1 / D) 2.2 \times 10^{-6},
$$

where $R_{\mathrm{s}}$ is in nanometer and $D$ is in centimeter squared per second.

Simply knowing, $R_{\mathrm{s}}$ is not very valuable in itself, except for estimating the degree of asymmetry, but this would be the same analysis developed above for $S_{\max } / S$. However, if one determines both $R_{\mathrm{s}}$ and $S$, this permits a direct determination of molecular weight, which cannot be deduced from either one alone. This is described in the next section.

\section{Determining the Molecular Weight of a Protein Molecule- Combining $S$ and $R_{\mathrm{S}}$ à la Siegel and Monte}

With the completion of multiple genomes and increasingly good annotation, the primary sequence of almost any protein can be found in the databases. The molecular weight of every protein subunit is therefore known from its sequence. But an experimental measure is still needed to determine if the native protein in solution is a monomer, dimer, or oligomer, or if it forms a complex with other proteins. If one has a purified protein, the molecular weight can be determined quite accurately by sedimentation equilibrium in the analytical ultracentrifuge. This technique has made a strong comeback with the introduction of the Beckman XL-A analytical ultracentrifuge. There are a number of good reviews $(14,15)$, and the documentation and programs that come with the centrifuge are very instructive. 
What if one does not have an XL-A centrifuge or the protein of interest is not purified? In 1966, Siegel and Monte (4) proposed a method that achieves the results of sedimentation equilibrium, with two enormous advantages. First, it requires only a preparative ultracentrifuge for sucrose or glycerol gradient sedimentation and a gel filtration column. This equipment is available in most biochemistry laboratories. Second, the protein of interest need not be purified; one needs only an activity or an antibody to locate it in the fractions. This is a very powerful technique and should be in the repertoire of every protein biochemist.

The methodology is very simple. The protein is run over a calibrated gel filtration column to determine $R_{\mathrm{s}}$ and hence $f$. Separately, the protein is centrifuged through a glycerol or sucrose gradient to determine $S$. One then uses the Svedberg equation (Eq. 4.1) to obtain $M$ as a function of $R_{\mathrm{s}}$ and $S$.

$$
M=S N_{o}\left(6 \pi \eta R_{s}\right) /\left(1-v_{2} \rho\right)
$$

setting $\eta=0.01, v_{2} \rho=0.73$, converting $S$ to Svedberg units and $R_{s}$ to nanometer, we can simplify further:

$$
M=4,205\left(S R_{s}\right)
$$

where $S$ is in Svedberg units, $R_{\mathrm{s}}$ is in nanometer, and $M$ is in Daltons.

This is pretty simple! Importantly, in typical applications, this method gives the protein mass within about $\pm 10 \%$. This is more than enough precision to distinguish between monomer, dimer, or trimer.

\footnotetext{
Application to SMC protein from B. subtilis. In the sections above, we showed how $S$ of the SMC protein from B. subtilis was determined to be $6.3 \mathrm{~S}$ from glycerol gradient sedimentation, and $R_{s}$ was $10.3 \mathrm{~nm}$, from gel filtration. Putting these values in Eq. 7.1b, we find that the molecular weight of SMC protein from B. subtilis is $273,000 \mathrm{Da}$. From the amino acid sequence, we know that the molecular weight of one SMC protein from B. subtilis subunit is 135,000 Da. The Siegel-Monte analysis finds that the SMC protein from B. subtilis molecule is a dimer.
}

Knowing that SMC protein from B. subtilis is a dimer with molecular weight 270,000 Da, we can now determine its $S_{\max } / S$. $S_{\max }$ is 15.1 (Eq. 4.3b) so $S_{\max } / S$ is 2.4. The SMC protein from B. subtilis molecule is thus expected to be highly elongated. EM (see below) confirmed this prediction.

\section{Electron \\ Microscopy of Protein Molecules}

Since the early 1980s, electron microscopy has become a powerful technique for determining the size and shape of single protein molecules, especially ones larger 
than $100 \mathrm{kDa}$. Two techniques available in most EM laboratories, rotary shadowing and negative stain, can be used for imaging single molecules. Cryo-EM is becoming a powerful tool for protein structural analysis, but it requires special equipment and expertise. For a large number of applications, rotary shadowing and negative stain provide the essential structural information.

For rotary shadowing, a dilute solution of protein is sprayed on mica, the liquid is evaporated in a high vacuum, and platinum metal is evaporated onto the mica at a shallow angle. The mica is rotated during this process, so the platinum builds up on all sides of the protein molecules. The first EM images of single protein molecules were obtained by Hall and Slayter using rotary shadowing (16). Their images of fibrinogen showed a distinctive trinodular rod. However, rotary shadowing fell into disfavor because the images were difficult to reproduce. Protein tended to aggregate and collect salt, rather than spread as single molecules. In 1976, James Pullman, a graduate student at the University of Chicago, then devised a protocol with one simple but crucial modificationhe added $30 \%$ glycerol to the protein solution. For reasons that are still not understood, the glycerol greatly helps the spreading of the protein as single molecules.

Pullman never published his protocol, but two labs saw his mimeographed notes and tested out the effect of glycerol, as a part of their own attempts to improve rotary shadowing (17, 18). They obtained reproducible and compelling images of fibrinogen (the first since the original Hall and Slayter study and confirming the trinodular rod structure) and spectrin (the first ever images of this large protein). The technique has since been used in characterizing hundreds of protein molecules.

Figure 4 shows rotary shadowed SMC protein from B. subtilis, fibrinogen, and hexabrachion (tenascin). SMC protein from B. subtilis is highly elongated, consistent with its high $S_{\max } / S$ discussed above (19). The fibrinogen molecules show the trinodular rod, but these images also resolved a small fourth nodule next to the central nodule (20), not seen in earlier studies. The central nodule is about $50 \mathrm{kDa}$, and the smaller fourth nodule is about $20 \mathrm{kDa}$. The "hexabrachion" tenascin molecule (21) illustrates the power of rotary shadowing at two extremes. First, the molecule is huge. Each of its six arms is made up of $\sim 30$ repeating small domains, totaling 200,000 Da. At the larger scale, the EM shows that each arm is an extended structure, matching the length expected if the repeating domains are an extended string of beads. At the finer scale, the EM can distinguish the different sized domains. The inner segment of each arm is a string of $3.5-\mathrm{kDa}$ epidermal growth factor domains, seen here as a thinner segment. A string of $10-\mathrm{kDa} F N-I I I$ domains is clearly distinguished as a thicker outer segment. The terminal knob is a single $22-\mathrm{kDa}$ fibrinogen domain. The $R_{\min }$ of these domains are $0.8,1.7$, and $2.8 \mathrm{~nm}$, and these can be distinguished by rotary shad- 


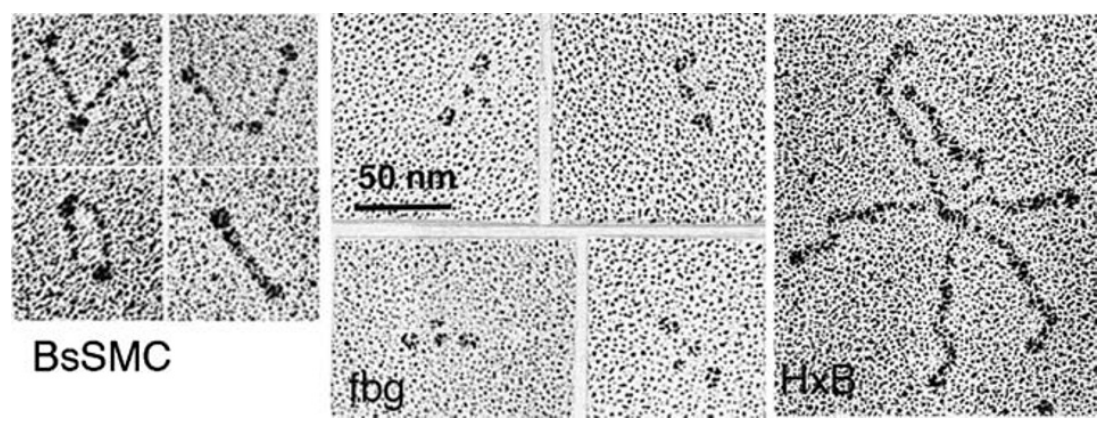

Fig. 4. Rotary shadowing EM of three highly elongated protein molecules: the SMC protein from $B$. subtilis (19), fibrinogen (20), and the hexabrachion protein, tenascin (21).

owing. Rotary shadowing EM can visualize single globular domains as small as $10 \mathrm{kDa}(3.5 \mathrm{~nm}$ diameter) and elongated molecules as thin as $1.5 \mathrm{~nm}$ (collagen).

Negative stain is another EM technique capable of imaging single protein molecules. It is especially useful for imaging larger molecules with a complex internal structure, which appear only as a large blob in rotary shadowing. Importantly, noncovalent protein-protein bonds are sometimes disrupted in the rotary shadowing technique (8), but uranyl acetate, in addition to providing high resolution contrast, fixes oligomeric protein structures in a few milliseconds (22). An excellent review of modern techniques of negative staining, with comparison to cryo-EM, is given in (23).

The simple picture of the molecule produced by EM is frequently the most straightforward and satisfying structural analysis at the $1-2-n m$ resolution. When the structure is confirmed by hydrodynamic analysis, the interpretation is even more compelling.

\section{Hydrodynamic Analysis and EM Applied to Large Multisubunit Complexes}

The text box above showed the application of the Siegel-Monte analysis to SMC protein from $B$. subtilis, which had only one type subunit and was found to be a dimer. Similar hydrodynamic analysis can be used to analyze multisubunit protein complexes. There are many examples in the literature; I will show here an elegant application to DASH/Daml.

The protein complex called DASH or Daml is involved in attaching chromosomal kinetochores to microtubules in yeast. $\mathrm{DASH} / \mathrm{Daml}$ is a complex of ten proteins that assemble into a particle containing one copy of each subunit. These complexes further assemble into rings that can form a sliding washer on the microtubule $(24,25)$. The basic ten-subunit complex has been purified from yeast and has also been expressed in Escheri- 
chia coli and purified (this required the heroic effort of expressing all ten proteins simultaneously (24)). Figure 5 shows the hydrodynamic characterization of the purified protein complex and illustrates several important features.

- For both the gel filtration (size exclusion chromatography, Fig. 5a) and gradient sedimentation, Fig. 5b, two calibration curves of known protein standards are shown, green and black. These are independent calibration runs. In this study, the gel filtration column was calibrated in terms of the reciprocal diffusion coefficient, $1 / D$, which is proportional to $R_{\mathrm{s}}$ (Eq. 6.2).

- The fractions were analyzed by Western blot for the location of two proteins of the complex, Spc34p and Hsk3p. Methods notes that $1 \mathrm{ml}$ fractions from gel filtration were precipitated with perchloric acid and rinsed with acetone prior to SDSPAGE, an essential amplification for the dilute samples of

a

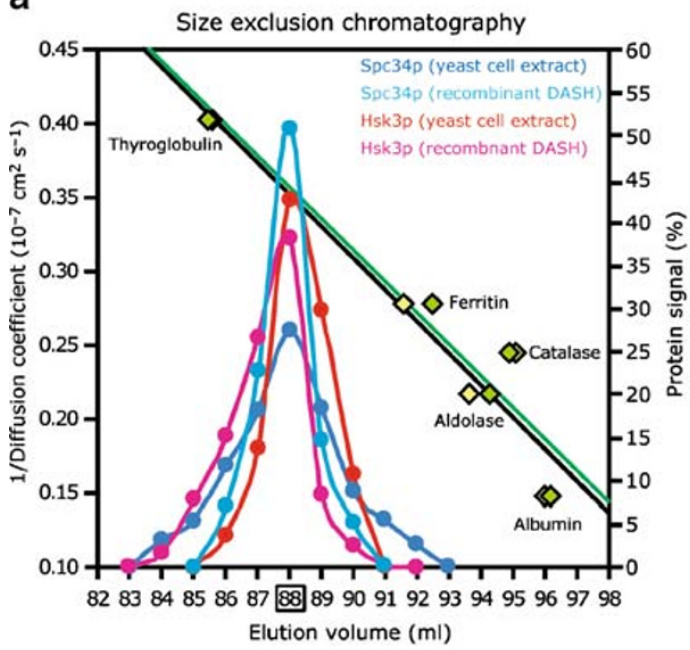

b

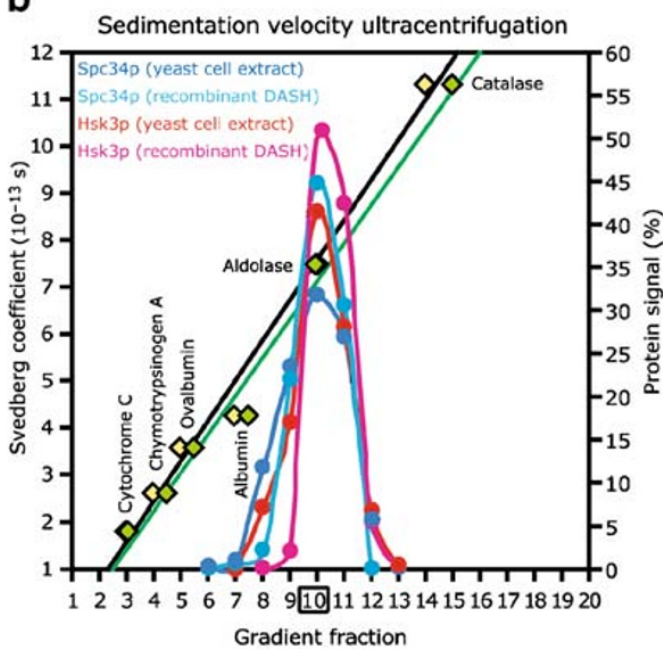

C

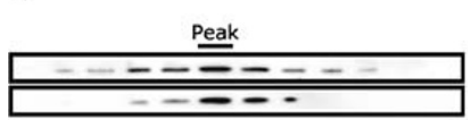

Yeast $\frac{\text { cell extract }}{\text { anti-Spc34p }}$ anti-Spc34p
anti-Hsk3p

Recombinant DASH

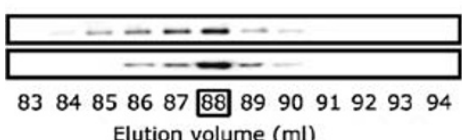
anti-Spc34p anti-Hsk3p

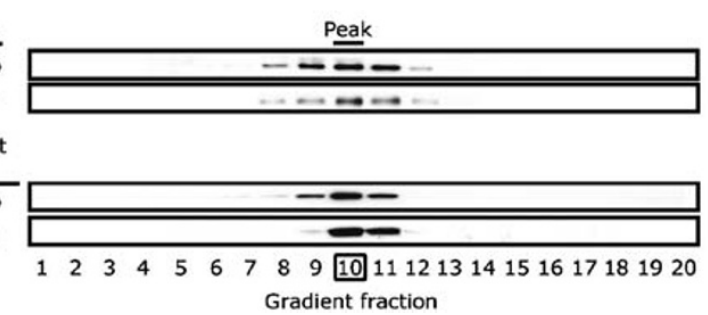

Fig. 5. Hydrodynamic analysis of the DASH/Dam1 complex. Gel filtration is shown in a and sucrose gradient sedimentation in $\mathbf{b}$. Independent calibration curves using standard proteins are shown in black and green. Dark and light blue show Spc34p in yeast cytoplasmic extract and in the purified recombinant protein. Red and purple show Hsk3p. The proteins were identified and quantitated by Western blot of the fractions, shown in c. The four protein bands eluted together at $1 / D=0.35 \times 10^{7}$, corresponding to $R_{\mathrm{s}}=7.6 \mathrm{~nm}$, and at 7.4 S. Reproduced from Miranda et al. (24) with permission of the authors. 
yeast cytoplasmic extract. These two proteins eluted together in both gel filtration and sedimentation, consistent with their being part of the same complex.

- The profiles of the two proteins were identical when analyzed in their native form in yeast cytoplasmic extract and as the purified complex expressed in E. coli. This is strong evidence that the expression protein is correctly folded and assembled.

- There is minimal trailing of any subunits. This means that there is no significant dissociation during the tens of minutes for the gel filtration, or the 12 -h centrifugation. The complex is held together by very high affinity bonds, making it essentially irreversible.

- Combining the $R_{\mathrm{s}}=7.6 \mathrm{~nm}$ (from $1 / D=0.35 \times 10^{-7}$, and $S=$ $7.4, \mathrm{Eq} \cdot 7.1 \mathrm{~b}$ gives a mass of $M=236 \mathrm{kDa}$, close to the $204 \mathrm{kDa}$ obtained from adding the mass of the ten subunits. $S_{\max }$ is 12.6 giving $S_{\max } / S=1.7$, suggesting a moderately elongated protein.

Figure 6 shows EM images of DASH/DAMl by rotary shadowing (a) and negative stain (b). Rotary shadowing showed irregular particles about $13 \mathrm{~nm}$ in diameter (24). The particles had variable and frequently elongated shapes, but internal structure could not be resolved. A later study used state of the art negative staining and sophisticated computer programs to sort images into classes and average them (26). These images resolved a complex internal structure. The negative stain showed most of the particles $(80 \%)$ to be dimers, with $15 \%$ monomers and $5 \%$ trimers. This contradicts the hydrodynamic analysis of Miranda et al. (24)
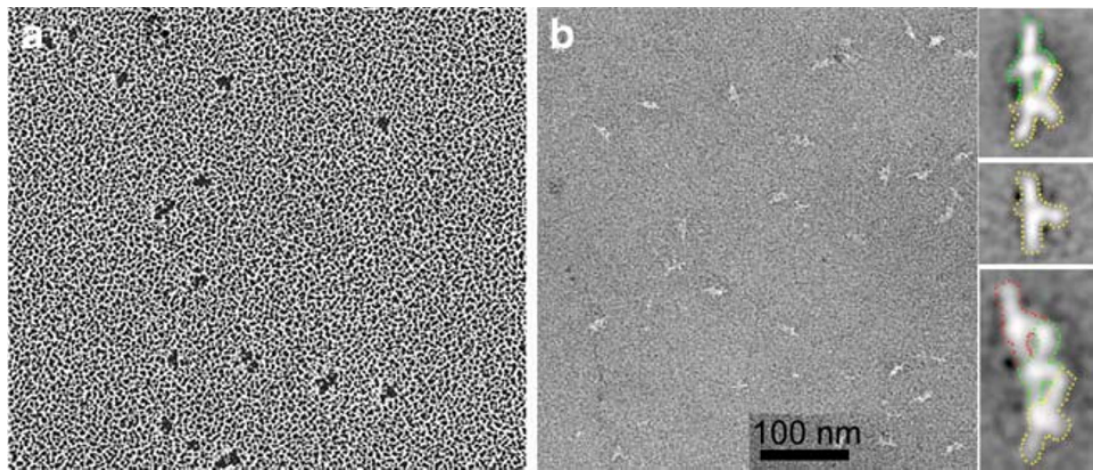

Fig. 6. EM of DASH/Dam1. a Rotary shadowing shows particles roughly $13 \mathrm{~nm}$ in size, with irregular shape. $\mathbf{b}$ State-of-the-art negative stain coupled with single particle averaging shows a complex internal structure of the elongated particles. The scale bar indicates $100 \mathrm{~nm}$ for the unprocessed images. The averaged images on the right show a monomer, dimer, and trimer. These panels are $14 \mathrm{~nm}$ wide. The dimer was the predominant species. Left panel (rotary shadowing) reprinted with permission of Miranda et al. (24). Right panels (negative stain) reprinted with permission of Wang et al. (26). 
showing that the particles were monomers. The reason for this discrepancy is not known.

\section{Acknowledgment}

\section{Supported by NIH grant CA47056.}

\section{References}

1. Richards F. M. (1974) The interpretation of protein structures: total volume, group volume distributions and packing density. J Mol Biol 82:1-14

2. Gittes F., Mickey B., Nettleton J., Howard J. (1993) Flexural rigidity of microtubules and actin filaments measured from thermal fluctuations in shape. J Cell Biol 120:923934

3. Sober H.A. (1966) Handbook of Biochemistry. The Chemical Rubber Co., Cleveland, $\mathrm{OH}$

4. Siegel L. M., Monte K. J. (1966) Determination of molecular weights and frictional ratios of proteins in impure systems by use of gel filtration and density gradient centrifugation. Biochim Biophys Acta 112:346362

5. Hesterberg L. K., Lee J. C., Erickson H. P. (1981) Structural properties of an active form of rabbit muscle phosphofructokinase. J Biol Chem 256:9724-9730

6. Teller D. C., Swanson E., De Haen C. (1979) The translational friction coefficient of proteins. Methods Enzymol 61:103-124

7. Garcia De La Torre J., Huertas M. L., Carrasco B. (2000) Calculation of hydrodynamic properties of globular proteins from their atomic-level structure. Biophys $J$ 78:719-30

8. Schürmann G., Haspel J., Grumet M., Erickson H. P. (2001) Cell adhesion molecule Ll in folded (horseshoe) and extended conformations. Mol Biol Cell 12:1765-73

9. Kirkwood J. G. (1954) The general theory of irreversible processes in solutions of macromolecules. J Polymer Sci 12:1-14

10. Bloomfield V., Dalton W. O., van Holde K. E. (1967) Frictional coefficients of multisubunit structures. I. Theory. Biopolymers 5:135-148

11. Carrasco B., Garcia de la Torre J. (1999) Hydrodynamic properties of rigid particles: comparison of different modeling and computational procedures. Biophys J 76:304457

12. Garcia de la Torre J., Llorca O., Carrascosa J. L., Valpuesta J. M. (2001) HYDROMIC: prediction of hydrodynamic properties of rigid macromolecular structures obtained from electron microscopy images. Eur Biophys J 30:457-62

13. Byron O. (2008) Hydrodynamic modeling: the solution conformation of macromolecules and their complexes. Methods Cell Biol 84:327-73

14. Schuster T. M., Toedt J. M. (1996) New revolutions in the evolution of analytical ultracentrifugation. Curr Opin Struct Biol 6:650-658

15. Hansen J. C., Lebowitz J., Demeler B. (1994) Analytical ultracentrifugation of complex macromolecular systems. [Review]. Biochemistry 33:13155-13163

16. Hall C. E., Slayter H. S. (1959) The fibrinogen molecule: its size, shape, and mode of polymerization. J Biophys Biochem Cytol 5:11-6

17. Fowler W. E., Erickson H. P. (1979) Trinodular structure of fibrinogen. Confirmation by both shadowing and negative stain electron microscopy. JMol Biol 134:241-249

18. Shotton D. M., Burke B. E., Branton D. (1979) The molecular structure of human erythrocyte spectrin. J Mol Biol 131:303329

19. Melby T. E., Ciampaglio C. N., Briscoe G., Erickson H. P. (1998) The symmetrical structure of structural maintenance of chromosomes (SMC) and MukB proteins: long, antiparallel coiled coils, folded at a flexible hinge. J Cell Biol 142:1595-1604

20. Erickson H. P., Fowler W. E. (1983) Electron microscopy of fibrinogen, its plasmic fragments and small polymers. Ann $N \Upsilon$ Acad Sci 408:146-163 
21. Erickson H. P., Iglesias J. L. (1984) A six-armed oligomer isolated from cell surface fibronectin preparations. Nature 311: 267-269

22. Zhao F. Q., Craig R. (2003) Capturing time-resolved changes in molecular structure by negative staining. J Struct Biol $141: 43-52$

23. Ohi M., Li Y., Cheng Y., Walz T. (2004) Negative staining and image classificationpowerful tools in modern electron microscopy. Biol Proced Online 6:23-34

24. Miranda J. J., De Wulf P., Sorger P. K., Harrison S. C. (2005) The yeast DASH complex forms closed rings on microtubules. Nat Struct Mol Biol 12:138-43

25. Westermann S., Avila-Sakar A., Wang H. W., Niederstrasser H., Wong J., Drubin D. G., Nogales E., Barnes G. (2005) Formation of a dynamic kinetochore-microtubule interface through assembly of the Daml ring complex. Mol Cell 17:277-90

26. Wang H. W., Ramey V. H., Westermann S., Leschziner A. E., Welburn J. P., Nakajima Y., Drubin D. G., Barnes G., Nogales E.
(2007) Architecture of the Daml kinetochore ring complex and implications for microtubule-driven assembly and forcecoupling mechanisms. Nat Struct Mol Biol 14:721-6

27. Aukhil I., Joshi P., Yan Y., Erickson H. P. (1993) Cell- and heparin-binding domains of the hexabrachion arm identified by tenascin expression proteins. $J$ Biol Chem 268:2542-2553

28. Leahy D. J., Axel R., Hendrickson W. A. (1992) Crystal structure of a soluble form of the human $\mathrm{T}$ cell coreceptor CD8 at 2.6 Å resolution. Cell 68:1145-1162

29. Chen S. C., Kramer G., Hardesty B. (1989) Isolation and partial characterization of an $\mathrm{Mr} 60,000$ subunit of a type $2 \mathrm{~A}$ phosphatase from rabbit reticulocytes. J Biol Chem 264:7267-75

30. Groves M. R., Hanlon N., Turowski P., Hemmings B. A., Barford D. (1999) The structure of the protein phosphatase $2 \mathrm{~A}$ PR65/A subunit reveals the conformation of its 15 tandemly repeated HEAT motifs. Cell 96:99-110 